\title{
Analysis of gestational trophoblastic disease by genotyping and chromosome in situ hybridization
}

\author{
Caroline YL Lai ${ }^{1}$, Kelvin YK Chan ${ }^{1,2}$, Ui-Soon Khoo ${ }^{1}$, Hextan YS Ngan ${ }^{2}$, Wei-Cheng Xue ${ }^{1}$, \\ Pui Man Chiu ${ }^{1}$, Sai-Wah Tsao ${ }^{3}$ and Annie NY Cheung ${ }^{1}$ \\ ${ }^{1}$ Department of Pathology; ${ }^{2}$ Department of Obstetrics and Gynaecology and ${ }^{3}$ Department of Anatomy, The \\ University of Hong Kong, Hong Kong, China
}

\begin{abstract}
Hydatidiform mole is classified into partial and complete subtypes according to histopathological and genetic criteria. Distinction between the two by histology alone may be difficult. Genetically, a complete mole is diploid without maternal contribution, whereas a partial mole is triploid with a maternal chromosome complement. To assess the accuracy of histological diagnosis by correlating with the genetic composition, we performed fluorescent microsatellite genotyping to detect the presence or absence of maternal genome in a hydatidiform mole and carried out chromosome in situ hybridization to analyze the ploidy. For genotyping analysis, paraffin sections of 36 complete and nine partial moles, diagnosed according to histological criteria, were microdissected and DNA was separately extracted from the decidua and molar villi. Six pairs of primers that flank polymorphic microsatellite repeat sequences on five different chromosomes were used. In all, 34 cases, including 31 complete moles and three partial moles diagnosed histologically, showed no maternal contribution by genotyping; thus these could be genetically considered as complete mole. The other 11 cases (five complete moles and six partial moles previously diagnosed by histology) showed the presence of maternal contribution and were genetically diagnosed as partial moles. The genotyping results correlated with histological evaluation in $88 \%$ (37/45) of hydatidiform mole and correlated with chromosome in situ hybridization findings in all the cases, that is, triploid hydatidiform moles had maternal-derived alleles, while diploid hydatidiform moles were purely androgenetic. Compared with genetic diagnosis, histological evaluation was more reliable for the diagnosis of a complete mole $(91 \%, 31 / 34)$ than that of a partial mole $(55 \%, 6 / 11)(P=0.0033)$. Seven complete moles and three partial moles diagnosed genetically developed gestational trophoblastic neoplasia. To conclude, genotyping and chromosome in situ hybridization can provide reliable adjunct to histology for the classification of a hydatidiform mole, especially in cases with difficult histological evaluation and early gestational age. As a partial mole still carries a risk of developing gestational trophoblastic neoplasia, follow-up is considered necessary for both complete and partial moles.
\end{abstract}

Modern Pathology (2004) 17, 40-48, advance online publication, 19 November 2003; doi:10.1038/modpathol.3800010

Keywords: hydatidiform mole; genotyping; chromosome in situ hybridization

Gestational trophoblastic disease is a disease of the trophoblastic tissue that includes a heterogeneous variety of lesions with variable degree of neoplastic changes, including hydatidiform mole, invasive mole, choriocarcinoma, placental site trophoblastic tumor, and epithelioid trophoblastic tumor. ${ }^{1,2}$

Hydatidiform mole is basically an abnormal conceptus characterized by significant hydropic enlargement and variable trophoblastic proliferation

Correspondence: Dr ANY Cheung, Department of Pathology, The University of Hong Kong, Queen Mary Hospital, Pokfulam Road, Hong Kong, China. E-mail: anycheun@hkucc.hku.hk

Received 17 June 2003; revised 20 August 2003; accepted 10 September 2003; published online 19 November 2003 involving part or all chorionic villi. Hydatidiform mole can be subclassified into complete and partial moles based on morphological, pathological, and genetic differences. ${ }^{3,4}$ The reported incidences of subsequent development into gestational trophoblastic neoplasia requiring chemotherapy following complete and partial moles were quite variable, affected by the referral patterns, diagnostic criteria, and criteria for post-evacuation chemotherapy. ${ }^{1,2,5,6}$ However, it is generally believed that such a risk is much higher after a complete mole when compared with a partial mole. The incidences of post-evacuation gestational trophoblastic neoplasia ranged from 8 to $29 \%$ in a complete mole and $0.5-5.5 \%$ in a partial mole. It is thus necessary to differentiate complete from partial moles. 
Traditionally, complete and partial moles are considered histologically different. Complete mole is a hydatidiform mole that rarely has any form of fetal development, with hydropic change of nearly all the villi, and generalized trophoblastic hyperplasia. On the other hand, partial mole has a certain degree of fetal development, with edematous change of a portion of the villi, and focal trophoblastic hyperplasia.

Genetically, complete and partial moles are two distinct entities. Complete moles are usually diploid, and almost all are androgenetic in origin, with all the 46 chromosomes originating entirely from the paternal side. ${ }^{7-9}$ On the other hand, most partial moles are triploid. ${ }^{3,4,7}$ They are formed by dispermic fertilization of a normal ovum. ${ }^{8,9}$ Thus, both complete and partial moles possess two sets of paternal haplotypes (diandric) in the genome. Nevertheless, unlike a complete mole, which came from an empty ovum with no maternal contribution, a partial mole has one set of chromosomes contributed by the maternal ovum.

The diagnosis of hydatidiform mole based on morphological differences alone has the problem of inter- and intra-observer variability. ${ }^{10-13}$ As a result, techniques that make use of the ploidy difference between complete and partial moles such as DNA flow or image cytometry, ${ }^{5,14-20}$ chromosome in situ hybridization, ${ }^{14,15,17}$ as well as polymerase chain reaction (PCR)-based microsatellite genotyping ${ }^{21-23}$ and HLA typing ${ }^{24}$ have been evolved to help in distinguishing complete from partial moles.

In this study, we used fluorescent microsatellite genotyping after microdissection to identify the parental origin of the molar tissue, which together with in situ hybridization, was used to assess the accuracy of histological differential diagnosis of complete and partial moles. The diagnosis was then correlated with the clinical diagnosis. The techniques can readily work on routinely processed evacuated tissue without risk of radioactive hazards, do not require extra blood samples from patient and spouse, and may function as useful adjunct to histological diagnosis.

\section{Materials and methods}

\section{Patients}

A total of 45 cases of hydatidiform mole were retrieved from the file of Department of Pathology, Queen Mary Hospital, the University of Hong Kong. The original pathology diagnoses were made between 1990 and 2000 based on histological criteria ${ }^{1,2}$ after examination of hematoxylin and eosin (H\&E) stained sections of each case. In all, 36 complete moles and nine partial moles were thus diagnosed. The diagnosis was extracted from the original pathology reports. Formalin-fixed, paraffin-embedded tissue blocks of these cases contained both maternal decidua and/or endometrium and molar villi. The patients' age ranged from 19 to 51 years, and the gestational age of these molar pregnancies estimated from the last menstrual period ranged from 6 to 37 weeks (Table 2).

\section{Clinical Follow-up}

After suction evacuation of the molar pregnancy, patients were monitored in a standard fashion including serial serum and urinary human chorionic gonadotrophin ( $\beta$-hCG) assays. $^{25}$ In our center, gestational trophoblastic neoplasia is suspected when $\beta$-hCG levels remained the same for 4 weeks or if there is rising $\beta$-hCG levels for three consecutive weeks when pregnancy is excluded. Patients with suspected gestational trophoblastic neoplasia are evaluated for evidence of metastatic disease. Eight of the 36 histologically diagnosed complete moles and two of the nine partial moles developed gestational trophoblastic neoplasia, and four of them developed metastasis (Table 2).

\section{DNA Preparation}

For each case, the maternal decidua and molar villi were separately microdissected from $6 \mu \mathrm{m}$ H\&E stained sections using the Arcturus PixCell ${ }^{\circledR}$ II LM 200 Laser Capture Microdissection System (Arcturus Engineering, Inc., Mountain View, CA, USA). Subsequently, DNA was prepared from the laser microdissected tissue according to the manufacturer's protocol. Briefly, DNA of the dissected cells on the Capsure ${ }^{\mathrm{TM}}$ HS LCM cap was extracted by bringing the cap surface into contact with a digestion buffer containing $0.04 \%$ proteinase $\mathrm{K}, 10 \mathrm{mM}$ Tris-HCl pH 8.0, $1 \mathrm{mM}$ EDTA, and 1\% Tween 20. For 500-1000 captured cells, $50 \mu \mathrm{l}$ of digestion buffer was required. After overnight incubation at $37^{\circ} \mathrm{C}$, the extraction tube was centrifuged for $5 \mathrm{~min}$ at a speed of less than $6400 \mathrm{rpm}(2000 \mathrm{~g})$ in order to collect the fluid containing DNA at the bottom of the tube. After verification of complete digestion of the cells by checking the cap under the microscope, the fluid was heated to $95^{\circ} \mathrm{C}$ for $10 \mathrm{~min}$ in order to inactivate the proteinase $\mathrm{K}$. A volume of $3 \mu \mathrm{l}$ of the fluid was then ready for use as a template for PCR.

\section{PCR Amplification}

In each case, $3 \mu \mathrm{l}$ of DNA from both maternal and molar tissues was amplified with six pairs of primers that flank polymorphic microsatellite repeat sequences on five different chromosomes (Table 1). The loci included D3S1358, D5S818, D13S267, D17S1322, D17S855, and vWA. PCR was performed in a $10 \mu \mathrm{l}$ reaction volume containing 1X PCR buffer (10 mM Tris-HCl, $50 \mathrm{mM} \mathrm{KCl} \mathrm{pH} \mathrm{8.3),} 2.0$ or $2.5 \mathrm{mM}$ magnesium chloride, $250 \mu \mathrm{M}$ of each dNTP, $0.4 \mu \mathrm{M}$ each of the forward and reverse primers, 0.6 $\mathrm{U}$ of 
Table 1 Microsatellite primers used in this study

\begin{tabular}{|c|c|c|c|c|}
\hline Locus & Location & Repeat & Heterozygosity & Primer sequence \\
\hline D13S267 & $13 q 12$ & Di & 0.69 & $\begin{array}{l}\text { GGCCTGAAAGGTATCCTC } \\
\text { TCCCACCATAAGCACAAG }\end{array}$ \\
\hline D17S1322 & $17 q 21$ & Di & 0.67 & $\begin{array}{l}\text { CTAGCCTGGGCAACAAACGA } \\
\text { GCAGGAAGCAGGAATGGAAC }\end{array}$ \\
\hline D17S855 & $17 q 21$ & Di & 0.82 & $\begin{array}{l}\text { GGATGGCCTTTTAGAAAGTGG } \\
\text { ACACAGACTTGTCCTACTGCC }\end{array}$ \\
\hline D5S818 & $5 q 22$ & Tetra & 0.74 & $\begin{array}{l}\text { GGGTGATTTTCCTCTTTGGT } \\
\text { TGATTCCAATCATAGCCACA }\end{array}$ \\
\hline D3S1358 & $3 p 21$ & Tetra & 0.78 & $\begin{array}{l}\text { ACTGCAGTCCAATCTGGGT } \\
\text { ATGAAATCAACAGAGGCTTG }\end{array}$ \\
\hline vWA & $12 \mathrm{p} 13$ to pter & Tetra & 0.80 & $\begin{array}{l}\text { CCCTAGTGGATAAGAATAATC } \\
\text { GGACAGATGATAAATACATAGGATGGATGG }\end{array}$ \\
\hline
\end{tabular}

References of the loci were obtained from the Genome Database (http://www.gdb.org).

AmpliTaq Gold ${ }^{\circledR}$ (Applied Biosystems, Foster City, CA, USA). One of each pair of primers was labeled with the fluorescent dye fluorescein (6-FAM), hexachlorofluorescein (HEX), or carboxytetramethylrhodamine (TAMRA). The PCR reaction consisted of an initial denaturation step at $95^{\circ} \mathrm{C}$ for $12 \mathrm{~min}$, followed by 39 cycles of denaturation at $94^{\circ} \mathrm{C}$ for $30 \mathrm{~s}$, annealing at $60^{\circ} \mathrm{C}$ for $1 \mathrm{~min}$, with extension at $72^{\circ} \mathrm{C}$ for $40 \mathrm{~s}$, and a final extension at $72^{\circ} \mathrm{C}$ for $10 \mathrm{~min}$ using the GeneAmp ${ }^{\circledR}$ PCR System 9600 (Applied Biosystems, Foster City, CA, USA). After amplification, $2.5 \mu \mathrm{l}$ of each PCR reaction product underwent electrophoresis in a $2 \%$ agarose gel to assess the yield.

\section{Microsatellite Genotyping}

The PCR product of each sample was then diluted according to the yield. After diluting with an appropriate volume of a mixture of formamide and blue dextran/EDTA containing a carboxy-x-rhodamine (ROX), an internal size standard, the PCR products were denatured at $95^{\circ} \mathrm{C}$, placed on ice to chill, and then separated by electrophoresis in a $5 \%$ denaturing polyacrylamide gel using the ABI PRISM 377 DNA Sequencer (Applied Biosystems, Foster City, USA). Data analysis and fragment sizing of the microsatellite polymorphism were performed using the ABI PRISM GeneScan ${ }^{\mathbb{R}}$ Analysis Software Version 3.1 and the Genotyper ${ }^{\circledR}$ Fragment Analysis Software Version 2.5 (Applied Biosystems, Foster City, CA, USA). The allele sizes of microsatellite polymorphisms found in the molar villi were then compared with those in the maternal tissue. The absence of maternal genome in the hydatidiform mole was indicated by the exclusion of maternal allele(s) in the fetal tissues. In each case, out of a total of six or seven microsatellite markers, at least one has to be informative before a definite conclusion can be made. Alleles in the complete or partial moles that were not found in the maternal tissues were considered as paternal. On the contrary, those cases with one or more maternal alleles definitely demonstrated by all microsatellite markers were considered as biparental.

\section{Chromosome In Situ Hybridization}

The ploidy of these 45 cases of hydatidiform mole was studied with chromosome in situ hybridization. DNA probes specific for the pericentromeric regions of chromosome 11 (D11Z1), chromosome 16 (D16Z1), Y chromosome, DYZ5 (American Type Culture Collection, Rockville, MD, USA), and chromosome X (pBamX5) (generous gift of Dr AHN Hopman) were used, ${ }^{26,27}$ All DNA probes were labeled with biotin 11-dUTP by standard nick translation method (Boehringer Mannheim, Indianapolis, IN, USA).

Paraffin sections of $5 \mu \mathrm{m}$ thickness were mounted on $2 \%$ aminopropyltriethoxysilane (TESPA)-coated slides. After deparaffinization, the slides were incubated with proteinase $\mathrm{K}(250-500 \mu \mathrm{g} / \mathrm{ml})$ and $0.1 \%$ Triton X-100 at $50^{\circ} \mathrm{C}$. The labeled probes were added to the hybridization $\operatorname{mix}(60 \%$ formamide, $10 \%$ dextran sulfate, $2 \times$ SSC) and applied to the tissue sections at probe concentrations of $1 \mathrm{ng} / \mu \mathrm{l}$ of the hybridization mixture. Denaturation was performed at $90^{\circ} \mathrm{C}$, followed by overnight hybridization at $37^{\circ} \mathrm{C}$ with intermittent agitation. Immunocytochemistry was performed using avidin, biotinylated mouse anti-avidin, rabbit anti-mouse peroxidase, and 3,3'diaminobenzidine (Dakopatts Ltd., UK) and hydrogen peroxidase to visualize peroxidase activity.

In each of the sections hybridized with individual, chromosome-specific DNA probes, at least 200 nuclei were assessed by independent observers (Xue and Cheung). A gain in chromosome 11 or 16 was inferred when $15 \%$ or more of the tumor cells displayed three or more than three signals.

\section{Data Analysis}

Correlation between the ploidy and histological diagnoses of the hydatidiform moles with results 
Table 2 Correlation between clinical parameters, histological diagnosis, and findings of genotyping and ploidy analysis

\begin{tabular}{|c|c|c|c|c|c|c|c|c|c|c|}
\hline \multirow[t]{2}{*}{ Case } & \multirow[t]{2}{*}{ Mat age } & \multirow[t]{2}{*}{ Gest age } & \multirow[t]{2}{*}{ Presence of mat alleles } & \multicolumn{2}{|c|}{$D x$ by } & \multirow[t]{2}{*}{$\mathrm{CISH}$} & \multicolumn{2}{|c|}{ Corr with } & \multirow[t]{2}{*}{ GTN } & \multirow[t]{2}{*}{ Metastasis } \\
\hline & & & & Histology & Genotype & & Histology & $\mathrm{CISH}$ & & \\
\hline 1 & 26 & 14 & Yes & PHM & PHM & $3 X$ & Yes & Yes & No & No \\
\hline 2 & 26 & 18 & No & CHM & CHM & $2 X$ & Yes & Yes & No & No \\
\hline 3 & 31 & NA & Yes & CHM & PHM & $3 X$ & No & Yes & Yes & No \\
\hline 4 & 37 & 6 & No & PHM & CHM & $2 \mathrm{X}$ & No & Yes & No & No \\
\hline 5 & 31 & 22 & No & CHM & CHM & $2 X$ & Yes & Yes & Yes & No \\
\hline 6 & 34 & 9 & Yes & CHM & PHM & $3 X$ & No & Yes & Yes & Yes \\
\hline 7 & 33 & NA & No & CHM & CHM & $2 X$ & Yes & Yes & No & No \\
\hline 8 & 28 & 12 & No & CHM & CHM & $2 X$ & Yes & Yes & No & No \\
\hline 9 & 30 & NA & Yes & CHM & PHM & $3 X$ & No & Yes & Yes & No \\
\hline 10 & 34 & NA & No & $\mathrm{CHM}$ & $\mathrm{CHM}$ & $2 \mathrm{X}$ & Yes & Yes & No & No \\
\hline 11 & 19 & 13 & No & CHM & CHM & $2 \mathrm{X}$ & Yes & Yes & No & No \\
\hline 12 & 35 & 6 & No & CHM & CHM & $2 X$ & Yes & Yes & No & No \\
\hline 13 & 27 & 11 & No & CHM & CHM & $2 X$ & Yes & Yes & Yes & Yes \\
\hline 14 & 45 & 16 & No & CHM & CHM & $2 X$ & Yes & Yes & Yes & Yes \\
\hline 15 & 51 & 16 & No & CHM & CHM & $2 X$ & Yes & Yes & No & No \\
\hline 16 & 21 & NA & No & CHM & CHM & $2 \mathrm{X}$ & Yes & Yes & No & No \\
\hline 17 & 45 & 14 & Yes & CHM & PHM & $3 X$ & No & Yes & No & No \\
\hline 18 & 26 & 12 & No & CHM & CHM & $2 X$ & Yes & Yes & No & No \\
\hline 19 & 31 & 9 & No & CHM & CHM & $2 X$ & Yes & Yes & Yes & No \\
\hline 20 & 29 & 20 & No & CHM & CHM & $2 X$ & Yes & Yes & No & No \\
\hline 21 & 37 & 37 & Yes & PHM & PHM & $3 X$ & Yes & Yes & No & No \\
\hline 22 & 38 & 16 & Yes & CHM & PHM & $3 X$ & No & Yes & No & No \\
\hline 23 & 31 & 9 & No & CHM & CHM & $2 X$ & Yes & Yes & No & No \\
\hline 24 & 25 & NA & No & CHM & CHM & $2 X$ & Yes & Yes & No & No \\
\hline 25 & 25 & 10 & No & CHM & CHM & $2 \mathrm{X}$ & Yes & Yes & No & No \\
\hline 26 & 29 & 20 & No & CHM & CHM & $2 \mathrm{X}$ & Yes & Yes & No & No \\
\hline 27 & 37 & 11 & No & CHM & CHM & $2 X$ & Yes & Yes & Yes & No \\
\hline 28 & 30 & 18 & No & CHM & CHM & $2 X$ & Yes & Yes & No & No \\
\hline 29 & 28 & NA & No & CHM & CHM & $2 X$ & Yes & Yes & No & No \\
\hline 30 & 17 & 7 & No & CHM & CHM & $2 X$ & Yes & Yes & No & No \\
\hline 31 & 32 & 14 & Yes & PHM & PHM & $3 X$ & Yes & Yes & No & No \\
\hline 32 & 33 & 13 & No & CHM & CHM & $2 X$ & Yes & Yes & No & No \\
\hline 33 & 24 & 10 & No & CHM & CHM & $2 \mathrm{X}$ & Yes & Yes & Yes & Yes \\
\hline 34 & 29 & 8 & Yes & PHM & PHM & $3 X$ & Yes & Yes & No & No \\
\hline 35 & 36 & 10 & Yes & PHM & PHM & $3 X$ & Yes & Yes & No & No \\
\hline 36 & 43 & 11 & No & CHM & CHM & $2 X$ & Yes & Yes & No & No \\
\hline 37 & 28 & 10 & No & CHM & CHM & $2 \mathrm{X}$ & Yes & Yes & No & No \\
\hline 38 & 23 & 16 & No & CHM & CHM & $2 \mathrm{X}$ & Yes & Yes & No & No \\
\hline 39 & 23 & 19 & No & $\mathrm{CHM}$ & CHM & $2 \mathrm{X}$ & Yes & Yes & No & No \\
\hline 40 & 34 & 11 & No & CHM & CHM & $2 X$ & Yes & Yes & No & No \\
\hline 41 & 34 & 8 & No & PHM & CHM & $2 X$ & No & Yes & No & No \\
\hline 42 & 45 & 8 & No & CHM & CHM & $2 \mathrm{X}$ & Yes & Yes & Yes & No \\
\hline 43 & 39 & 7 & No & PHM & CHM & $2 X$ & No & Yes & No & No \\
\hline 44 & 32 & 10 & No & CHM & CHM & $2 \mathrm{X}$ & Yes & Yes & No & No \\
\hline 45 & 35 & 15 & Yes & PHM & PHM & $3 X$ & Yes & Yes & No & No \\
\hline
\end{tabular}

Mat, maternal; Gest, gestational; Dx, Diagnosis; Corr, correlation; NA, Not available; CHM, complete mole; PHM, partial mole.

of genotyping was made. Statistical analysis was performed with Fisher's Exact Test using the Statistical Package for Social Science (PC window version 8). A histological review was carried out in those cases with discrepancy between the original histological diagnosis and the genetic findings.

\section{Results}

\section{Fluorescent Microsatellite Genotyping}

The details of genotyping used to deduce the absence or presence of maternal genome in 45 cases of hydatidiform mole were presented in Table 1 . Based on the above criteria for evaluation of the genotyping results, 34 cases seem to indicate an absence of maternal contribution (Figure 1). These include 31 complete moles and three partial moles diagnosed histologically. On the other hand, findings in 11 cases were consistent with a biparental origin (Figure 2). Of these, there were five histologically diagnosed complete moles and six partial moles.

Out of the 34 cases of diploid hydatidiform moles, $22(65 \%)$ cases were homozygous for a single allele, which suggests that those hydatidiform moles 
probably resulted from homozygous (monospermic) fertilization of an enucleated ovum by a single haploid sperm followed by endoreduplication of the paternal genome. For the remaining 12 cases (35\%), the diploid hydatidiform moles were found to be

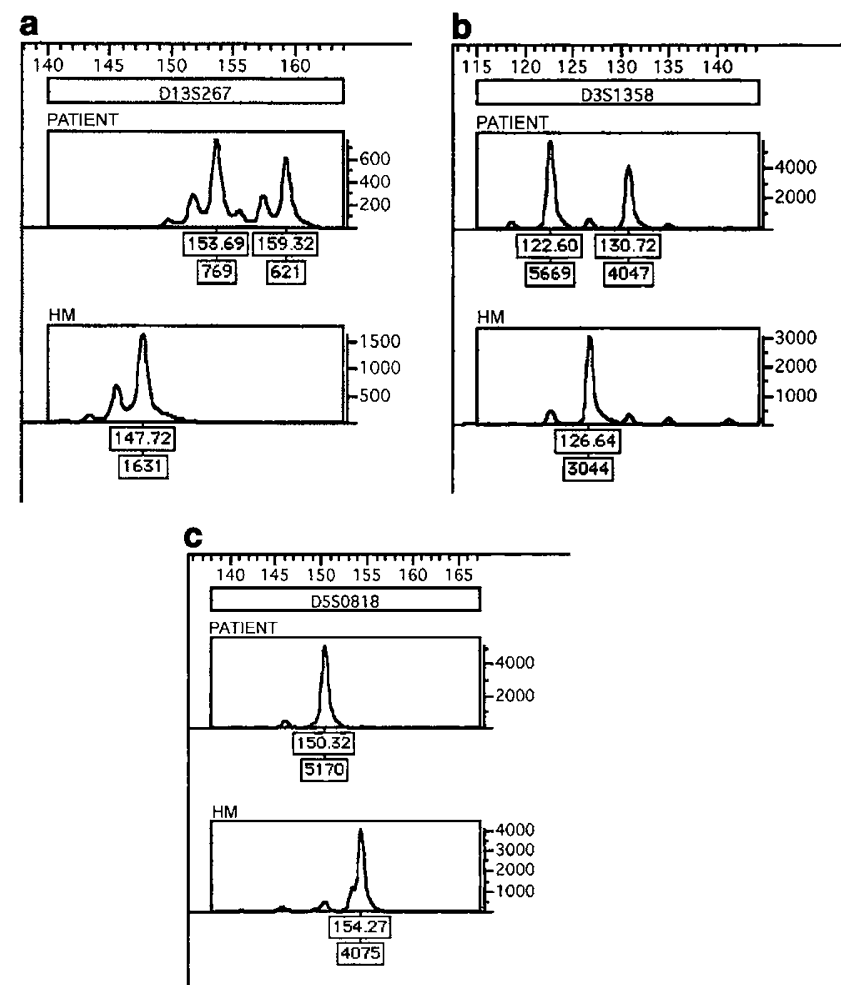

Figure 1 Microsatellite polymorphisms of the patient, and the diploid hydatidiform moles (HM) in Case 27. The patient was heterozygous for the markers (a) D13S267 and (b) D3S1358, homozygous for (c) D5S818, generating 154-160, 123-131 and $150 \mathrm{bp}$ alleles, respectively. The hydatidiform mole was homozygous, giving rise to alleles 148,127 , and 154 , which were not found in the maternal DNA.
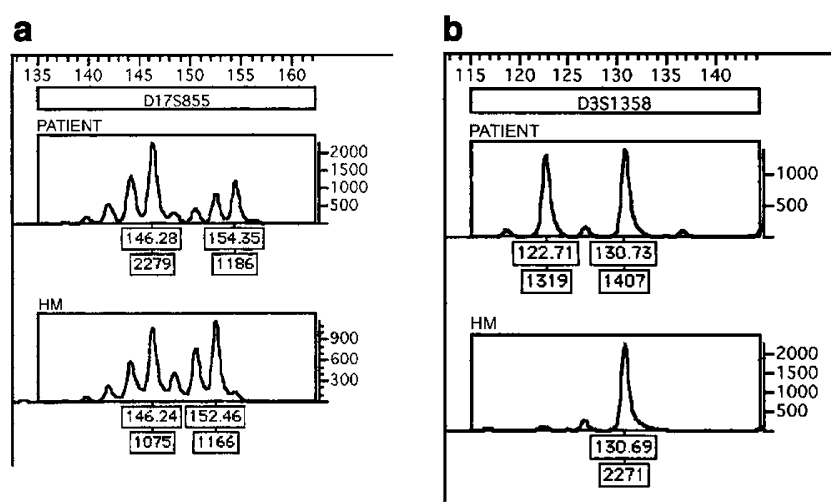

Figure 2 Microsatellite polymorphisms of the patient, and the triploid hydatidiform moles (HM) in Case 34. The patient was heterozygous for the markers (a) D17S855 and (b) D3S1358, generating alleles of $147-155$ and $123-131 \mathrm{bp}$, respectively. The hydatidiform mole was heterozygous for D17S855, homozygous for D3S1358, giving rise to alleles 147-153 and 131. The maternal alleles of sizes 147 and $131 \mathrm{bp}$ were present. homozygous for some, but heterozygous for other alleles. Hence, those cases were thought to be arising from heterozygous (dispermic) fertilization of an enucleate egg by two different haploid sperms.

\section{Histology}

According to previously issued pathology reports, nine of the 45 samples of hydatidiform mole studied were diagnosed as partial moles (Table 2). The diagnosis was made based on the presence of a certain degree of fetal development, absence of florid hyperplasia of the trophoblasts, and hydropic swelling in some but not all villi (Figure 3). ${ }^{1,2} \mathrm{~A}$ fetus was identified in one partial mole (Case 45). Fetal membrane was found in Case 21, while microscopic examination of the tissues demonstrated the presence of nucleated red blood cells in one case (Case 31). The remaining 36 cases were classified as complete moles (Table 2) based on the histological criteria such as the absence of any fetal development, conspicuous trophoblastic hyperplasia, and generalized hydropic swelling of nearly all the placental villi. ${ }^{1,2}$

\section{Chromosome In Situ Hybridization}

Chromosome in situ hybridization was performed on all the 45 cases of hydatidiform mole. The results of chromosome in situ hybridization showed that out of the 36 histologically diagnosed complete moles, 31 (86\%) displayed disomy (Figure 4), while five $(14 \%)$ demonstrated trisomy (Figure 5) in the chromosome copy number studied (Table 2). For the nine histologically diagnosed partial moles, six $(67 \%)$ were trisomic and three (33\%) were found to be disomic. The results of the above chromosome

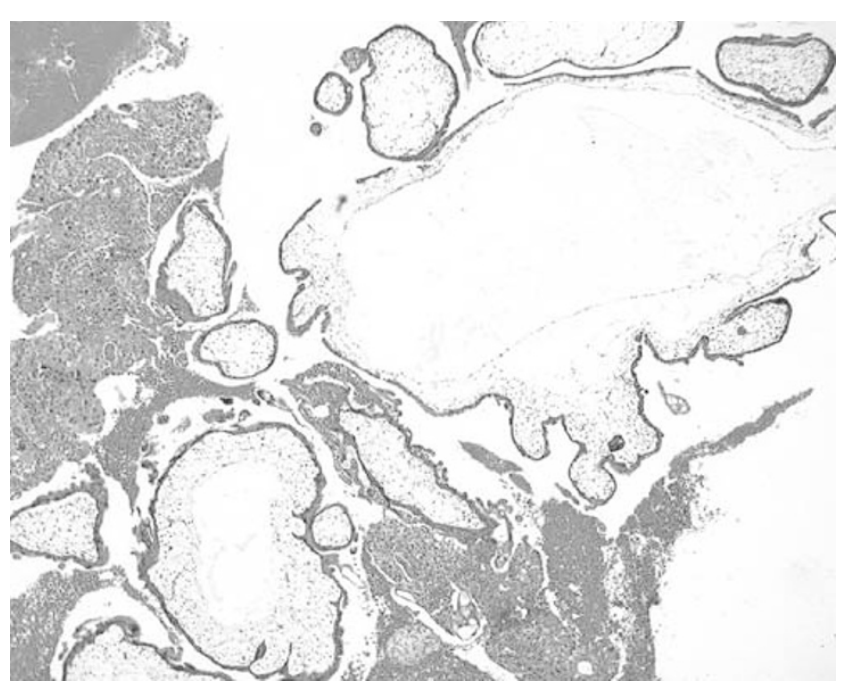

Figure 3 Photomicrograph of a partial mole (Case 34) with relatively normal-sized villi and hydropic villi with cistern formation (hematoxylin and Eosin, $\times 80$ ). 
in situ hybridization and genotyping experiments were compatible with each other in all the cases.

\section{Correlation Between Histological Diagnosis and Genotyping}

Genotyping results correlated with histology in $82 \%$ (37/45) of cases, being more consistent in complete moles $(91 \%, 31 / 34)$ than in partial moles $(55 \%, 6 /$ 11) $(P=0.0033)$ (Table 3$)$. Genotyping findings in all the cases correlated with the chromosome in situ hybridization findings. Triploid hydatidiform moles had maternally derived alleles, while diploid hydatidiform moles were purely androgenetic. The results of genotyping of two cases selected from

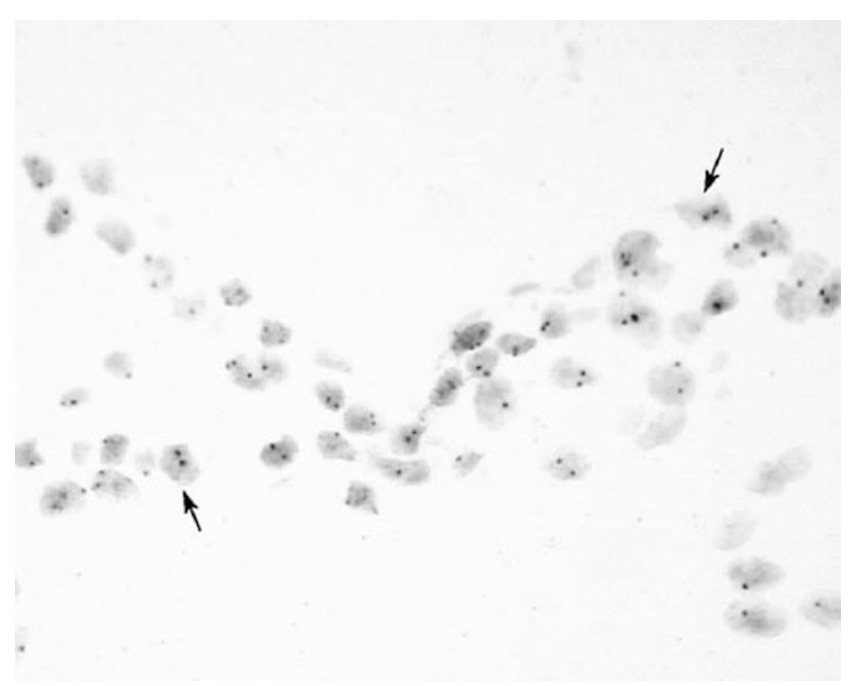

Figure 4 Two hybridization signals were found in the majority of the nuclei of Case 27 as detected by DNA probes for chromosome 16.

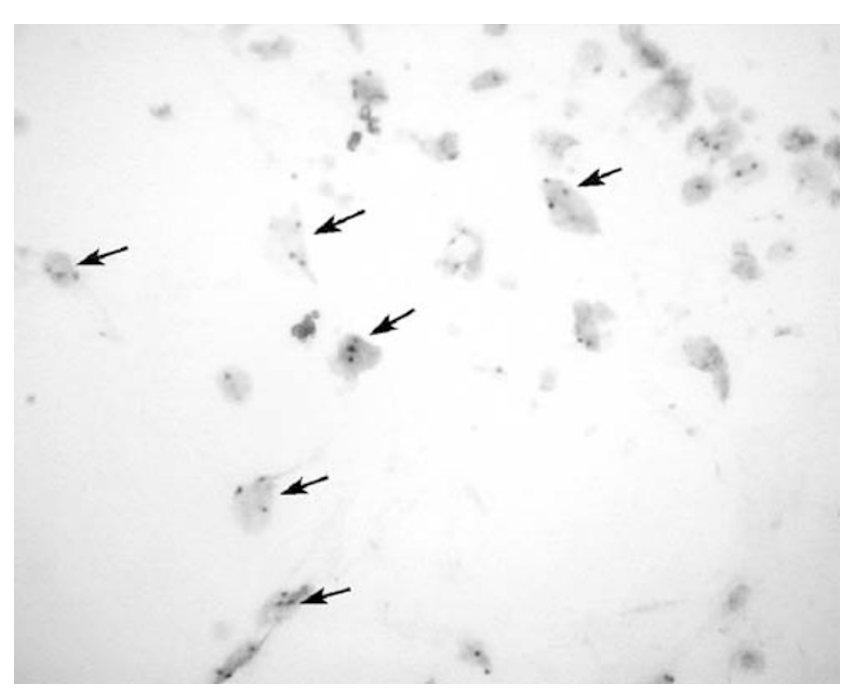

Figure 5 Three or more hybridization signals were found in the majority of the nuclei of Case 34 as detected by DNA probes for chromosome 16 . complete moles and partial moles are illustrated in Figures 1 and 2.

Three genotypically complete moles were diagnosed as partial moles histologically in this study. All three cases were diagnosed at an early gestational age (6-8 weeks), and the histological features of partial moles were not yet well established. Reviewed histology was compatible with complete moles. Five genotypically classified partial moles were diagnosed as complete moles histologically. Two cases were diagnosed at second trimester with conspicuous cistern formation and prominent trophoblastic proliferation in some of the villi. Normalsized villi can be identified after review of all the histology slides, and these five cases were histologically compatible with partial moles.

\section{Discussion}

Traditionally, diagnosis of hydatidiform mole is often made on the basis of the morphological differences between complete and partial moles. However, classification of hydatidiform mole according to histological features alone has the problem of inter- and intra-observer variability. ${ }^{10-13}$ In early evacuated complete moles (first 5-9 weeks of gestation), when the distinct features of villous edema and trophoblastic hyperplasia have not been fully developed, the histological diagnosis between complete and partial moles may be particularly difficult and complete moles may be misdiagnosed as partial moles. ${ }^{11,28,29}$ Partial moles may also be underdiagnosed as hydropic abortion or misdiagnosed as complete moles. ${ }^{11,28-31}$ Even the presence of amnion, or other fetal tissues, associated with molar tissue should not always be considered indicative of a diagnosis of partial moles since these tissues could be of androgenetic origin related to the existence of early embryonic development in complete moles. ${ }^{32}$

Since complete and partial moles are genetically distinct, several techniques that make use of these differences have been developed to help improve accuracy in diagnosis. Cytogenetic analysis, DNA flow cytometry, and chromosome in situ hybridization are useful for ploidy determination of the molar tissue. Hydatidiform moles could be subclassified

Table 3 Correlation between histological diagnosis and genotyping of hydatidiform mole

\begin{tabular}{lrcc}
\hline Genotyping & \multicolumn{3}{c}{ Histological diagnosis } \\
\cline { 2 - 4 } & CHM & PHM & \\
\hline CHM & 31 & 3 & 34 \\
PHM & 5 & 6 & 11 \\
& 36 & 9 &
\end{tabular}

$P=0.0033$. 
into complete and partial moles according to histological and cytogenetic criteria. ${ }^{3,4,7}$ Most partial moles were found to be triploid, whereas complete moles were diploid.

Cytogenetic analysis by karyotyping requires fresh tissue for cell culture and preparation of metaphase spreads. In situations where fresh tissue is not available for karyotyping, ploidy determination can be achieved by DNA flow cytometry and chromosome in situ hybridization. Both methods can be applied to fixed, paraffin-embedded tissues. The distinct difference of diploid and triploid cell populations found in complete and partial moles, respectively, can be detected easily. Chromosome in situ hybridization also helps to differentiate between XX and XY complete moles. ${ }^{27}$

The androgenetic origin of complete moles was revealed by Kajii and Ohama ${ }^{33}$ in 1977 based on chromosomal polymorphism. This finding was further supported by other groups. ${ }^{16,34}$ Most of the complete moles are monospermic and arise from fertilization of an empty egg by a haploid sperm followed by duplication of the sperm genome, ${ }^{35}$ resulting in a homozygous 46 , XX karyotype. The 46, YY karyotype is not viable. Some of the complete moles are dispermic, resulting from entry of two sperms into the empty ovum (heterozygous). ${ }^{36}$ It has been estimated that about $10-25 \%$ of complete moles are products of dispermy $y^{37-39}$ and genetically may be either heterozygous 46 , XX or $46, \mathrm{XY} \cdot{ }^{27}$ In the current study, about one-third of the diploid complete moles were heterozygous.

Although cytogenetic and ploidy analyses can usually differentiate a complete mole from a partial mole in most cases, some uncertainty can still exist, especially in some atypical cases. Therefore, molecular genetic techniques that can identify the parental origin of the fetal genome help to distinguish complete from partial moles, and heterozygous from homozygous complete moles. Such techniques may make use of the unique DNA polymorphisms in each individual, including restriction fragment length polymorphism (RFLP), ${ }^{38,39}$ variable number tandem repeat (VNTR), ${ }^{40}$ and microsatellite analysis ${ }^{32}$ can be used to determine the genetic origin of the hydatidiform mole.

In this study, we used fluorescent microsatellite genotyping in addition to histology, and chromosome in situ hybridization to classify 45 hydatidiform moles. Using a panel of seven microsatellite primers, these 36 complete moles and nine partial moles diagnosed according to histological criteria were reclassified. For hydatidiform moles, which were classified histologically as complete moles or nine partial moles, 37 (82\%) matched with the classification deduced from genotyping. Histological diagnosis is more reliable in the diagnosis of complete moles (91\%) than partial moles (55\%) $(P=0.0033)$. This observation concurs with the findings from previous studies that differential diagnosis of complete and partial moles based on histology alone can be difficult in some cases. ${ }^{10-13}$ On the other hand, all the results of chromosome in situ hybridization correlated with genotyping in that all the genotyped complete moles were diploid, while the genotyped partial moles were found to be triploid.

Three genotypically complete moles were initially histologically diagnosed as partial moles in this study. All three cases were diagnosed at early gestational age (6-8 weeks), and the histological features of partial moles were not well established, rendering the initial histological diagnosis difficult. On the other hand, there were five genotypically classified partial moles that were originally diagnosed as complete moles histologically. The conspicuous cistern formation and florid trophoblastic proliferation in a portion of the hydropic villi may mislead to the diagnosis of complete moles.

Earlier studies have demonstrated that most gestational trophoblastic neoplasia resulting from histologically diagnosed partial moles were in fact complete moles according to diploid DNA patterns. ${ }^{14}$ On the other hand, some partial moles, although rarely, did cause persistent disease $\mathrm{e}^{31,41-43}$ or even choriocarcinoma. ${ }^{44}$ Follow-up is still mandatory in cases of partial moles.

In this study, two-thirds of complete moles were found to be homozygous arising for duplication from a single spermatic haplotype. Actually, complete moles have been used as homozygosity reference for single-nucleotide polymorphism (SNP) analysis since the complete mole genotypes represent the haplotypes of their genomes arising from a duplicated set of chromosomes. ${ }^{45,46}$

To conclude, our findings support the use of molecular techniques as an adjunct to histological diagnosis of hydatidiform mole ${ }^{47}$ Genotyping using microsatellite analysis can reliably determine the presence or absence of maternal contribution to the molar genome using formalin-fixed, paraffin-embedded tissues of molar villi and decidua, even when paternal tissue is not available for evaluation. This method is useful in the routine classification of hydatidiform moles since it can be achieved readily with fluorescent microsatellite analysis using an automated DNA sequencer and is thus of clinical significance. Moreover, it allows retrospective study on archival material. The application of such molecular techniques should be encouraged in cases with difficulty in histological diagnosis. We also found that histological diagnosis is more reliable in a complete mole. Clinical follow-up of partial moles is still important since some of them will develop into gestational trophoblastic neoplasia requiring chemotherapy.

\section{Acknowledgements}

This study was supported by the HKSAR Research Grant Council Grant (10203228.13396.21200.324.01) and the University of Hong Kong Conference and 
Research Grant. We thank Mr Tony Chan for his technical advices in microdissection.

\section{References}

1 Mazur MT, Kurman RJ. Gestational trophoblastic disease. In: Kurman RJ (ed). Blaustein's Pathology of Female Genital Tract. Springer-Verlag: New York, 1994, pp 1049-1093.

2 Elston CW. Gestational trophoblastic disease. In: Fox H (ed). Haines and Taylor: Obstetrical and Gynaecological Pathology. Churchill Livingstone: Edinburgh, 1995, pp 1597-1640.

3 Szulman AE, Surti U. The syndromes of hydatidiform mole. II. Morphologic evolution of the complete and partial mole. Am J Obstet Gynecol 1978;132:20-27.

4 Szulman AE, Surti U. The syndromes of hydatidiform mole. I. Cytogenetic and morphologic correlations. Am J Obstet Gynecol 1978;131:665-671.

5 Paradinas FJ, Browne P, Fisher RA, et al. A clinical, histopathological and flow cytometric study of 149 complete moles, 146 partial moles and 107 non-molar hydropic abortions. Histopathology 1996;28:101-110.

6 Bagshawe KD, Lawler SD, Paradinas FJ, et al. Gestational trophoblastic tumours following initial diagnosis of partial hydatidiform mole. Lancet 1990;335:1074-1076.

7 Vassilakos P, Riotton G, Kajii T. Hydatidiform mole: two entities. A morphologic and cytogenetic study with some clinical consideration. Am J Obstet Gynecol 1977;127:167-170.

8 Lawler SD, Fisher RA, Pickthall VJ, et al. Genetic studies on hydatidiform moles. I. The origin of partial moles. Cancer Genet Cytogenet 1982;5:309-320.

9 Jacobs PA, Szulman AE, Funkhouser J, et al. Human triploidy: relationship between parental origin of the additional haploid complement and development of partial hydatidiform mole. Ann Hum Genet 1982;46:223-231.

10 Conran RM, Hitchcock CL, Popek EJ, et al. Diagnostic considerations in molar gestations. Hum Pathol 1993;24:41-48.

11 Sumithran E, Cheah PL, Susil BJ, et al. Problems in the histological assessment of hydatidiform moles: a study on consensus diagnosis and ploidy status by fluorescent in situ hybridisation. Pathology 1996;28:311-315.

12 Messerli ML, Parmley T, Woodruff JD, et al. Inter- and intra-pathologist variability in the diagnosis of gestational trophoblastic neoplasia. Obstet Gynecol 1987;69:622-626.

13 Howat AJ, Beck S, Fox H, et al. Can histopathologists reliably diagnose molar pregnancy? J Clin Pathol 1993;46:599-602.

14 Van de Kaa CA, Schijf CP, de Wilde PC, et al. Persistent gestational trophoblastic disease: DNA image cytometry and interphase cytogenetics have limited predictive value. Mod Pathol 1996;9:1007-1014.

15 Cheville JC, Greiner T, Robinson RA, et al. Ploidy analysis by flow cytometry and fluorescence in situ hybridization in hydropic placentas and gestational trophoblastic disease. Hum Pathol 1995;26:753-757.

16 Hemming JD, Quirke P, Womack C, et al. Diagnosis of molar pregnancy and persistent trophoblastic disease by flow cytometry. J Clin Pathol 1987;40: 615-620.
17 Van de Kaa CA, Hanselaar AG, Hopman AH, et al. DNA cytometric and interphase cytogenetic analyses of paraffin-embedded hydatidiform moles and hydropic abortions. J Pathol 1993;170:229-238.

18 Fisher RA, Lawler SD, Ormerod MG, et al. Flow cytometry used to distinguish between complete and partial hydatidiform moles. Placenta 1987;8: 249-256.

19 Fukunaga M. Flow cytometric and clinicopathologic study of complete hydatidiform moles with special reference to the significance of cytometric aneuploidy. Gynecol Oncol 2001;81:67-70.

20 Lage JM, Driscoll SG, Yavner DL, et al. Hydatidiform moles. Application of flow cytometry in diagnosis. Am J Clin Pathol 1988;89:596-600.

21 Lane SA, Taylor GR, Ozols B, et al. Diagnosis of complete molar pregnancy by microsatellites in archival material. J Clin Pathol 1993;46:346-348.

22 Abeln EC, Cornelisse CJ, Dreef EJ, et al. Molecular identification of a partial hydatidiform mole. Diagn Mol Pathol 1997;6:58-63.

23 Fisher RA, Newlands ES. Rapid diagnosis and classification of hydatidiform moles with polymerase chain reaction. Am J Obstet Gynecol 1993;168:563-569.

24 Bateman AC, Hemmatpour SK, Theaker JM, et al. Genetic analysis of hydatidiform moles in paraffin wax embedded tissue using rapid, sequence specific PCR-based HLA class II typing. J Clin Pathol 1997;50:288-293.

25 Wong LC, Ngan HY, Cheng DK, et al. Methotrexate infusion in low-risk gestational trophoblastic disease. Am J Obstet Gynecol 2000;183:1579-1582.

26 Xue WC, Guan XY, Ngan HY, et al. Malignant placental site trophoblastic tumor: a cytogenetic study using comparative genomic hybridization and chromosome in situ hybridization. Cancer 2002;94:2288-2294.

27 Cheung AN, Sit AS, Chung LP, et al. Detection of heterozygous XY complete hydatidiform mole by chromosome in situ hybridization. Gynecol Oncol 1994;55:386-392.

28 Bell KA, Van DV, Addya K, et al. Molecular genetic testing from paraffin-embedded tissue distinguishes nonmolar hydropic abortion from hydatidiform mole. Mol Diagn 1999;4:11-19.

29 Van de Kaa CA, Schijf CP, de Wilde PC, et al. The role of deoxyribonucleic acid image cytometric and interphase cytogenetic analyses in the differential diagnosis, prognosis, and clinical follow-up of hydatidiform moles. A report from the Central Molar Registration in The Netherlands. Am J Obstet Gynecol 1997;177: 1219-1229.

30 Jacobs PA, Hassold TJ, Matsuyama AM, et al. Chromosome constitution of gestational trophoblastic disease. Lancet 1978;2:49.

31 Surti U, Szulman AE, O’Brien S. Complete (classic) hydatidiform mole with 46,XY karyotype of paternal origin. Hum Genet 1979;51:153-155.

32 Lawler SD, Fisher RA, Dent J. A prospective genetic study of complete and partial hydatidiform moles. Am J Obstet Gynecol 1991;164:1270-1277.

33 Kajii T, Ohama K. Androgenetic origin of hydatidiform mole. Nature 1977;268:633-634.

34 Fisher RA, Povey S, Jeffreys AJ, et al. Frequency of heterozygous complete hydatidiform moles, estimated by locus-specific minisatellite and Y chromosome-specific probes. Hum Genet 1989;82: 259-263. 
35 Jacobs PA, Wilson CM, Sprenkle JA, et al. Mechanism of origin of complete hydatidiform moles. Nature 1980;286:714-716.

36 Kovacs BW, Shahbahrami B, Tast DE, et al. Molecular genetic analysis of complete hydatidiform moles. Cancer Genet Cytogenet 1991;54:143-152.

37 Weaver DT, Fisher RA, Newlands ES, et al. Amniotic tissue in complete hydatidiform moles can be androgenetic. J Pathol 2000;191:67-70.

38 Van de Kaa CA, Nelson KA, Ramaekers FC, et al. Interphase cytogenetics in paraffin sections of routinely processed hydatidiform moles and hydropic abortions. J Pathol 1991;165:281-287.

39 Fujita N, Tamura S, Shimizu N, et al. Genetic origin analysis of hydatidiform mole and non-molar abortion using the polymerase chain reaction method. Acta Obstet Gynecol Scand 1994;73:719-725.

40 Jeffers MD, O’Dwyer P, Curran B, et al. Partial hydatidiform mole: a common but underdiagnosed condition. A 3-year retrospective clinicopathological and DNA flow cytometric analysis. Int J Gynecol Pathol 1993;12:315-323.
41 Sunde L, Mogensen B, Olsen S, et al. Flow cytometric DNA analyses of 105 fresh hydatidiform moles, with correlations to prognosis. Anal Cell Pathol 1996;12: 99-114.

42 Fukunaga M. Early partial hydatidiform mole: prevalence, histopathology, DNA ploidy, and persistence rate. Virchows Arch 2000;437:180-184.

43 Szulman AE, Ma HK, Wong LC, et al. Residual trophoblastic disease in association with partial hydatidiform mole. Obstet Gynecol 1981;57:392-394.

44 Seckl MJ, Fisher RA, Salerno G, et al. Choriocarcinoma and partial hydatidiform moles. Lancet 2000;356:36-39.

45 Fan JB, Surti U, Taillon-Miller P, et al. Paternal origins of complete hydatidiform moles proven by whole genome single-nucleotide polymorphism haplotyping. Genomics 2002;79:58-62.

46 Taillon-Miller P, Piernot EE, Kwok PY. Efficient approach to unique single-nucleotide polymorphism discovery. Genome Res 1999;9:499-505.

47 Petignat $\mathrm{P}$, Billieux MH, Blouin JL, et al. Is genetic analysis useful in the routine management of hydatidiform mole? Hum Reprod 2003;18:243-249. 